\title{
Truly Conical Eleven Antenna: a New Geometry for Applications at High Frequencies
}

\author{
Jian Yang \\ Dept. of Signals and Systems \\ Chalmers University of Technology, Sweden \\ jian.yang@chalmers.se
}

\author{
Miroslav Pantaleev \\ Onsala Space Observatory, \\ Chalmers University of Technology, Sweden \\ miroslav.pantaleev@chalmers.se
}

\begin{abstract}
A new truly conical geometry of Eleven antenna for high frequency applications is proposed in the paper. By using this geometry, the antenna petal arrays are made on only one piece of flat thin PCB, which is soft enough to be conically bent and strong enough after the conical bending to be fastened stably by only the center pack cone and the outer walls. Therefore, many manufacture difficulties by using previous Eleven antenna geometries have been avoided. Simulated performance of a 4.620GHz Eleven feed, $-10 \mathrm{~dB}$ reflection coefficient and above $60 \%$ aperture efficiency when feeding a reflector antenna, is presented as an example in the paper.
\end{abstract}

\section{INTRODUCTION}

The Eleven antenna has many unique characteristics over ultra-wide frequency band [1]-[3], and therefore, found many applications in radio telescopes [4], [5] and potentially in other fields, such as surveillance applications.

Previous Eleven feeds were made by four tilted flat petals of cascaded log-periodic folded dipole arrays, which were either etched on printed circuit boards (PCBs) or pure metal structures for low-frequency antennas with dielectric support walls underneath.

For high frequencies, such as the Band B of 4.6-24 GHz in the SKA (square kilometer array) project, the tilted structure makes the fabrication of Eleven antenna a big challenge. High operation frequency of the Eleven antenna requires thin substrate board in PCB to avoid strong surface wave prorogating in the substrate, since the surface wave deteriorates the radiation performance. However, if a thin PCB is used, a thicker and even extra dielectric support walls are needed in order to make a stable tilted petals, where the thicker and extra dielectric walls will cause the same surface wave problem.

In this paper, a new solution, the conical structure of the Eleven antenna petal made on a flat thin PCB, is proposed to the problem mentioned above. The detailed structure of the proposed antenna and its advantages are discussed in Sect. II and simulated results with an example of $4.6-20 \mathrm{GHz}$ conical Eleven feed is presented in Sect. III.

\section{Truly Conical Structure}

We proposed a quasi-conical structure for circular Eleven feed in [3], where four flat PCBs formed an inverse pyramid with curved folded dipole arrays. When the operation frequency of the Eleven feed is high, the PCBs for the antenna petals are required to be thin in order to avoid surface

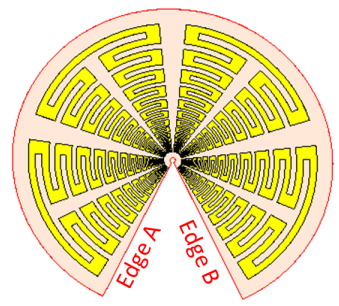

(a)

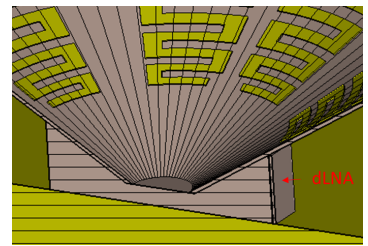

(c)

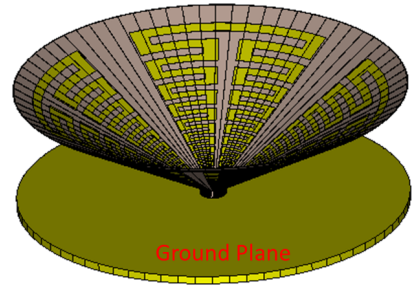

(b)

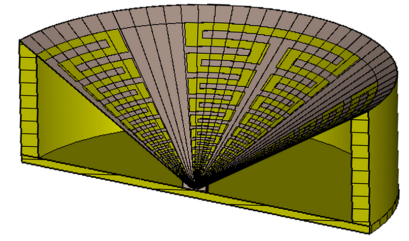

(d)
Fig. 1. Geometry of new truly conical Eleven antenna: (a) flat PCB (printed circuit board) of folded dipole array; (b) making of conical petal by a conical bending with edges A and B connected; (c) center pack with differential LNAs for feeding; (d) simple whole geometry. Note a half cutting is used in (c) and (d) for clarity.

wave propagation in substrate. This leads to that the thin PCBs are not mechanically hard enough to keep the flatness by themselves when the PCBs are tilted. In order to have the better flatness, more support walls are demanded, which then will cause surface wave prorogating along the walls. Therefore, a dilemma exists.

On the other hand, if we change our perspective to this thin PCB, just because of the thin substrate, the PCB is possible to be conically bent for many dielectric materials, and when the PCB cone has been made, the conical structure is mechanically much more stable than its flat state due to the elasticity and tension of the thin substrate, which presents an advantage of thin PCBs. We make use of this property to establish a new structure of the Eleven antenna.

Fig. 1 shows the procedure of making the conical Eleven array petals. Four log-periodic circular folded dipole arrays are etched on a thin flat PCB, where a part of circular substrate is cut away with edges A and B, shown in Fig. 1(a). Making the petal array cone by a conical bending with edges $\mathrm{A}$ and $\mathrm{B}$ connected, as shown in Fig. 1(b). The connection of edges A 


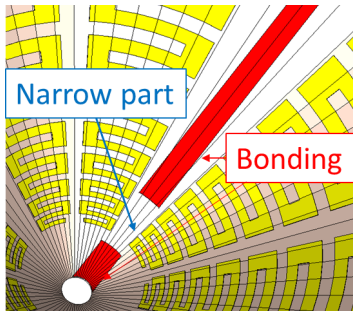

(a)

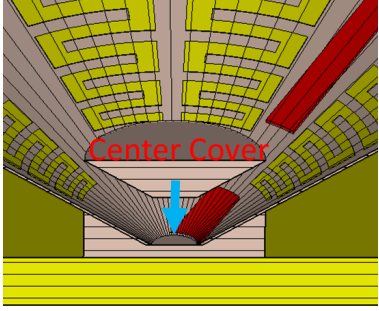

(b)
Fig. 2. Detail structures: (a) bonding structure and (b) center pack.

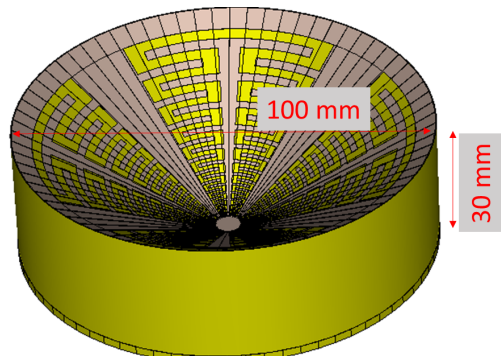

Fig. 3. $\quad 4.6-20 \mathrm{GHz}$ conical Eleven feed.

and $\mathrm{B}$ is a key issue in this new design. The connection can be achieved by thread bonding, glue bonding, combination of the two, or other technologies. Since the bonding is between folded dipole arrays, the effect of the bonding material on the electrical performance of the antenna is small. Note that actually it is not required that the bonding should be applied along the whole edges A and B. Fig. 2(a) shows one example of bonding area, where the narrow part does not need bonding, which makes the bonding easier. The petal cone is supported by a center pack and an outer metal wall, as shown in Figs. 1(c) and 1(d). A cover is used to strengthen the support at center pack (Fig. 2(b)). By this way, underneath dielectric support walls are avoided, and surface wave prorogation is suppressed.

The feeding for the conical Eleven feed is preferred by using active baluns - the differential low-noise amplifiers (dLNAs). Four dLNAs should be used in the feed, as shown in Fig. 1(d). Note that the active balun feeding here is just a proposal, we have not done any test or simulation for this feeding.

\section{Simulated Results}

Fig. 3 shows an example of conical Eleven feed: $4.6-20 \mathrm{GHz}$ feed. The PCB is $0.1 \mathrm{~mm}$ thick Rogers RO4350B substrate. The simulated reflection coefficient is shown in Fig. 4 and the aperture efficiency of the feed for a reflector with $2 \times 60^{\circ}$ subtended angle is in Fig. 5. Note that the dimensions of the 4.6-20GHz feed is determined by experience and have not gone through optimization. Nevertheless, the performance is very promising.

\section{CONCLUSIONS}

A new structure for Eleven antenna, the conical Eleven antenna made by a thin flat PCB, is proposed in the paper. This structure is attractive for Eleven antenna applications at high frequencies.

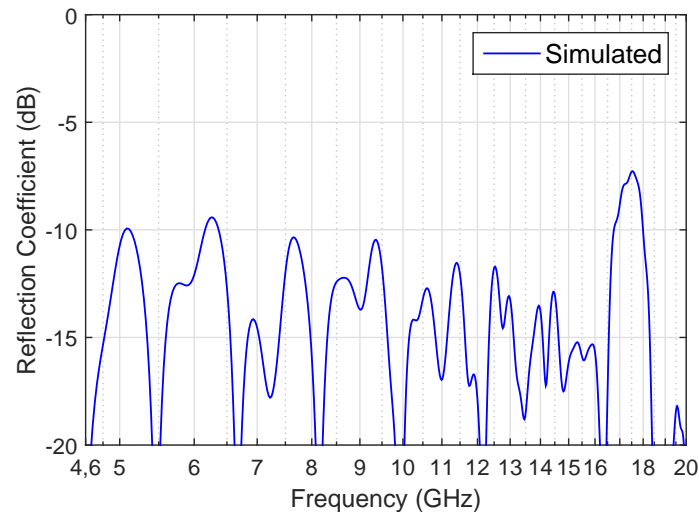

Fig. 4. Simulated reflection coefficient with $300 \mathrm{ohm}$ differential impedance at the input of each petal.

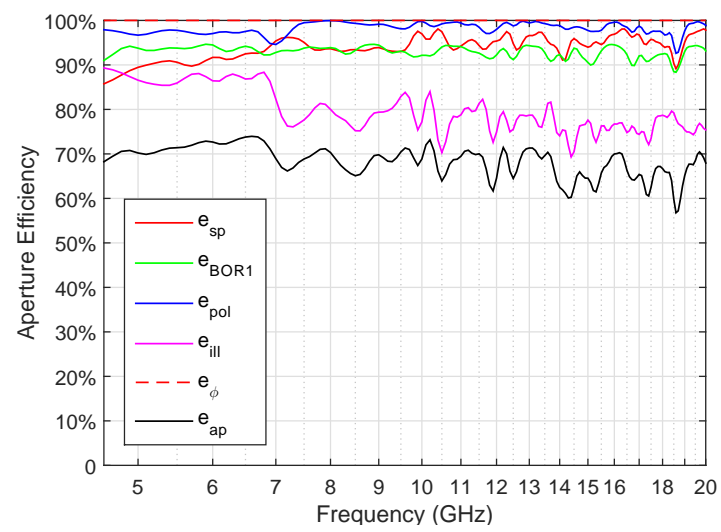

Fig. 5. Simulated aperture efficiency of the $4.6-20 \mathrm{GHz}$ feed when feeing a primary reflector with subtended angle of $2 \times 60^{\circ}$.

\section{ACKNOWLEDGEMENT}

This work has been supported by Swedish VR Research Infrastructures Planning Grant Swedish contributions to the SKA radio-telescope in its pre-construction phase (Contract No. C0546801).

\section{REFERENCES}

[1] R. Olsson, P.-S. Kildal, and S. Weinreb, "The Eleven antenna: a compact low-profile decade bandwidth dual polarized feed for reflector antennas," Antennas and Propagation, IEEE Transactions on, vol. 54, no. 2, pp. 368-375, 2006.

[2] J. Yang, M. Pantaleev, P. Kildal, B. Klein, Y. Karandikar, L. Helldner, N. Wadefalk, and C. Beaudoin, "Cryogenic 2-13 GHz Eleven feed for reflector antennas in future wideband radio telescopes," IEEE Transactions on Antennas and Propagation, vol. 59, no. 6, pp. 1918-1934, 2011.

[3] J. Yin, J. Yang, M. Pantaleev, and L. Helldner, "The circular Eleven antenna: a new decade-bandwidth feed for reflector antennas with a high aperture efficiency," IEEE Transactions on Antennas and Propagation, vol. 61, 2013.

[4] J. Yang, S. Pivnenko, and P.-S. Kildal, "Comparison of two decadebandwidth feeds for reflector antennas: the eleven antenna and quadridge horn," in Proc. 4th European Conf. Antennas and Propagation (EuCAP2010), 2010.

[5] J. Yang, M. Pantaleev, B. Billade, M. Ivashina, T. Carozzi, L. Helldner, and M. Dahlgren, "A compact dual-polarized 4-port eleven feed with high sensitivity for reflectors over 0.35-1.05 ghz," Antennas and Propagation, IEEE Transactions on, vol. 63, no. 12, pp. 5955-5960, 2015. 